\title{
Reversal time of the magnetization of single-domain ferromagnetic particles with mixed uniaxial and cubic anisotropy
}

\author{
William T. Coffey, ${ }^{1}$ Pierre-Michel Déjardin, ${ }^{2}$ and Yuri P. Kalmykov ${ }^{2}$ \\ ${ }^{1}$ Department of Electronic and Electrical Engineering, Trinity College, Dublin 2, Ireland \\ ${ }^{2}$ Laboratoire de Mathématiques, Physique et Systèmes, Université de Perpignan Via Domitia, \\ 52, Avenue Paul Alduy, 66860 Perpignan Cedex, France
}

(Received 26 March 2008; revised manuscript received 27 November 2008; published 2 February 2009)

\begin{abstract}
The reversal time of the magnetization of single-domain ferromagnetic particles is estimated for mixed uniaxial and cubic anisotropy energies possessing nonparaboloidal saddles and well bottoms or either. The calculation generalizes the existing adaptation of the Kramers escape rate theory to fine ferromagnetic particles with nonaxially symmetric magnetocrystalline-Zeeman energies, originally based on the paraboloidal approximation for the energy near its stationary points, yielding in addition a simple universal Kramers turnover formula (based on the Mel'nikov-Meshkov depopulation factor) for the reversal time valid for all values of the damping. The asymptotic solution is compared with the appropriate numerically exact solution of the corresponding Fokker-Planck equation for the probability density function of magnetization orientations. The predictions of the generalized nonparaboloidal stationary point turnover formula agree with the numerical solution for a wide range of damping and other parameters characterizing the mixed anisotropy.
\end{abstract}

DOI: 10.1103/PhysRevB.79.054401

PACS number(s): 75.60.Jk, 75.50.Tt, 76.20.+q, 05.40.-a

\section{INTRODUCTION}

Fine single-domain ferromagnetic particles are characterized by thermal instability of the magnetization ${ }^{1} \mathbf{M}(t)$ resulting in superparamagnetism ${ }^{2}$ because each behaves in a magnetic sense as a giant Langevin paramagnet. The thermal fluctuations and relaxation of $\mathbf{M}(t)$ currently merit attention in information storage and rock magnetism. The initial treatment of the thermal fluctuations due to Néel ${ }^{1}$ based on classical transition state theory (TST) was further developed by Brown. ${ }^{3,4} \mathrm{He}$ set the theory in the context of the classical Brownian motion, which unlike TST accounts for the departure from thermal equilibrium due to the energy interchange between a particle and its heat bath, by deriving the FokkerPlanck equation for the probability density function $W$ of magnetization orientations on a sphere of radius $M_{s}$, where $M_{s}$ is the saturation magnetization assumed constant so that the only variable is the orientation of $\mathbf{M}$. This equation is ${ }^{4}$

$$
2 \tau_{N} \frac{\partial W}{\partial t}=\frac{\beta}{\alpha} \mathbf{n} \cdot(\nabla V \times \nabla W)+\nabla \cdot(\nabla W+\beta W \nabla V),
$$

where $\nabla$ is the gradient operator on the unit sphere, $\mathbf{n}$ is a unit vector along $\mathbf{M}, V$ is the free-energy density comprising the nonseparable Hamiltonian of the anisotropy and Zeeman energy densities, $\beta=v /(k T), v$ is the volume of the ferromagnetic particle, $k$ is Boltzmann's constant, $T$ is the absolute temperature, $\alpha$ is a dimensionless damping constant, $\tau_{N}$ $=\tau_{0}\left(\alpha+\alpha^{-1}\right), \tau_{0}=\beta M_{s} /(2 \gamma)$ is the characteristic free diffusion time of $\mathbf{M}(t)$, and $\gamma$ is the gyromagnetic ratio.

One of the most important physical parameters is the magnetization reversal time $\tau$ due to thermal agitation over the internal magnetocrystalline energy barrier of the particle. In order to estimate $\tau$, Brown adapted to magnetization relaxation an ingenious method of Kramers ${ }^{5}$ for the thermally activated escape from a potential well of point Brownian particles of a single degree of freedom with separable and additive Hamiltonians. When the escape rates $\left(\sim \tau^{-1}\right)$ for these particles are calculated by the asymptotic Kramers method, three regimes of damping appear as a consequence of the asymptotic procedure, viz., (i) intermediate-to-high damping (IHD), (ii) very low damping (VLD), and (iii) turnover region. Kramers ${ }^{5}$ obtained so-called IHD and VLD formulas for the escape rate. However, he emphasized that he could not find a general method of attack in order to obtain an asymptotic formula valid for all damping regimes. This proposition known as the Kramers turnover problem was solved much later by Mel'nikov and Meshkov. ${ }^{6,7}$ They obtained a universal formula for the escape rate valid for all values of the damping by postulate from heuristic reasoning, essentially appealing to continuity, that a universal escape rate formula may be given by simply multiplying the IHD escape rate by a depopulation factor bridging the VLD and IHD solutions. By extending the Mel'nikov-Meshkov approach, Grabert ${ }^{8}$ and Pollak et al. ${ }^{9}$ later presented a complete solution of the Kramers turnover problem and showed that the Mel'nikov and Meshkov universal formula can be obtained without $a d$ hoc assumptions. The results of Mel'nikov and Meshkov, ${ }^{6}$ however, do not apply without modification to the escape rate of the magnetization; it differs fundamentally from point Brownian particles because $\mathbf{M}(t)$ has two degrees of freedom, namely, polar angles $(\vartheta$ and $\varphi)$, the undamped equation of motion is the gyromagnetic equation rather than Newton's equation, the Hamiltonian is inherently nonseparable, and the concept of inertia is meaningless. Nevertheless, inertia is essentially mimicked for nonaxially symmetric potentials only by the gyromagnetic term causing coupling or entanglement of the transverse and longitudinal modes of $\mathbf{M}(t)$. Hence, the familiar Kramers damping regimes may still occur in the asymptotic magnetization escape rate formulas, reflecting the fact that the transverse dynamics affects the longitudinal dynamics. This analogy allowed Smith and de Rozario ${ }^{10}$ and Brown ${ }^{4}$ to derive the magnetization escape rate in the IHD limit ${ }^{11}$ following the original Kramers method. Thus, in order to evaluate the IHD escape 
TABLE I. Range of validity of asymptotic formulas for $\Gamma_{i j}$.

\begin{tabular}{lcccc}
\hline \hline Escape rate & $\Gamma_{i j}^{\mathrm{TST}}$, Eq. (4) & $\Gamma_{i j}^{\mathrm{IHD}}$, Eq. (3) & $\Gamma_{i j}^{\mathrm{VLD}}$, Eq. (5) & $\Gamma_{i j}$, Eq. (6) \\
\hline Range of validity & $\alpha \approx 1$ & $\alpha \geq 1$ & $\alpha \ll 1$ & All $\alpha$ \\
References & 1 & 4 & 14 and 15 & 11 and 17 \\
\hline \hline
\end{tabular}

rate from a potential well $i$ to a well $j$, they supposed that the free-energy density $V(\mathbf{M})$ has a multistable structure with minima at $\mathbf{n}_{i}$ and $\mathbf{n}_{j}$ separated by a potential barrier at a saddle point $\mathbf{n}_{0}$. If $\mathbf{M}$ is close to a stationary point $\mathbf{n}_{k}[k$ $=(0, i, j)]$ and $\left(n_{1}^{(k)}, n_{2}^{(k)}, n_{3}^{(k)}\right)$ denote the direction cosines of $\mathbf{M}$, then $V(\mathbf{M})$ may be approximated by

$$
V \approx V_{k}+c_{1}^{(k)}\left(n_{1}^{(k)}\right)^{2} / 2+c_{2}^{(k)}\left(n_{2}^{(k)}\right)^{2} / 2,
$$

where $c_{1}^{(k)}=\partial^{2} V / \partial n_{1}^{(k) 2}$ and $c_{2}^{(k)}=\partial^{2} V / \partial n_{2}^{(k) 2}$. By substituting Eq. (2) into the Fokker-Planck equation (1), the latter may be solved following the Kramers method ${ }^{5}$ in the vicinity of the stationary points yielding the escape rate $\Gamma_{i j}^{\mathrm{IHD}}$ from well $i$ to well $j$ as 4,10

$$
\Gamma_{i j}^{\mathrm{IHD}}=\Gamma_{i j}^{\mathrm{TST}} \Omega_{0}(\alpha) / \omega_{0},
$$

with $\Gamma_{i j}^{\mathrm{TST}}$ as the escape rate for TST as applied to the magnetization, namely,

$$
\Gamma_{i j}^{\mathrm{TST}}=\frac{\omega_{i}}{2 \pi} e^{-\Delta V},
$$

where $\Delta V=\beta\left(V_{0}-V_{i}\right)$ is the dimensionless barrier height, $\omega_{i}=\gamma \sqrt{c_{1}^{(i)} c_{2}^{(i)}} / M_{s}$ and $\omega_{0}=\gamma \sqrt{-c_{1}^{(0)} c_{2}^{(0)}} / M_{s}$ are the well and saddle angular frequencies, respectively, and

$$
\begin{aligned}
\Omega_{0}(\alpha)= & \frac{\beta}{4 \tau_{0}\left(\alpha+\alpha^{-1}\right)}\left[\sqrt{\left(c_{2}^{(0)}-c_{1}^{(0)}\right)^{2}-4 \alpha^{-2} c_{1}^{(0)} c_{2}^{(0)}}\right. \\
& \left.-c_{1}^{(0)}-c_{2}^{(0)}\right]
\end{aligned}
$$

is the damped saddle angular frequency. Equation (3) is simply a special case of Langer's extension ${ }^{12}$ of the Kramers IHD escape rate to many degrees of freedom and nonseparable Hamiltonians in order to generalize the calculation of Becker and Döring ${ }^{13}$ of the rate of condensation of a supersaturated vapor.

Now for vanishing damping, $\alpha \rightarrow 0$, the IHD escape rate $\Gamma_{i j}^{\mathrm{IHD}}$ from Eq. (3) reduces to the TST escape rate $\Gamma_{i j}^{\mathrm{TST}}$, which is obviously independent of $\alpha$. However, by analogy with point particles ${ }^{11}$ this is not the true VLD limit or energy controlled diffusion, where the energy loss per cycle of the almost periodic motion of the magnetization on the saddlepoint energy (escape) trajectory is much less than the thermal energy, as noted by Klik and Gunther. ${ }^{14,15}$ Rather, it comprises $^{11}$ the intermediate damping limit corresponding to Néel's TST result. Recognizing this Klik and Gunther ${ }^{14,15}$ derived the correct VLD magnetization Kramers escape rate $\Gamma_{i j}^{\mathrm{VLD}}$, viz., ${ }^{11,14,15}$

$$
\Gamma_{i j}^{\mathrm{VLD}} \sim \alpha S_{i} \Gamma_{i j}^{\mathrm{TST}}
$$

where $S_{i}$ is the dimensionless action at the saddle-point energy (see Sec. II). The conditions of applicability of these IHD and VLD solutions for superparamagnets are defined by $\alpha \geq 1$ and $\alpha<0.01$, respectively. However, experimental values of $\alpha$ usually lie in the Kramers turnover region characterized by $10^{-2} \leq \alpha<1 .{ }^{16}$ Hence, Coffey et al. ${ }^{11}$ and Déjardin et al. ${ }^{17}$ extended the Mel'nikov-Meshkov formalism ${ }^{6,7}$ to the relaxation time of the magnetization. They obtained for the escape rate $\Gamma_{i j}$ from a single well ${ }^{11,17}$

$$
\Gamma_{i j}=A\left(\alpha S_{i}\right) \Gamma_{i j}^{\mathrm{IHD}}=A\left(\alpha S_{i}\right) \frac{\omega_{i} \Omega_{0}(\alpha)}{2 \pi \omega_{0}} e^{-\Delta V},
$$

where the magnetization depopulation factor $A$ is

$$
A(\delta)=\exp \left[\frac{1}{\pi} \int_{0}^{\infty} \frac{\ln \left\{1-\exp \left[-\delta\left(\lambda^{2}+1 / 4\right)\right]\right\}}{\lambda^{2}+1 / 4} d \lambda\right],
$$

the dimensionless action at the saddle-point energy $S_{i}$ is defined as

$$
S_{i}=\beta \oint_{V=V_{0}}\left(1-z^{2}\right) \frac{\partial V}{\partial z} d \varphi-\frac{1}{1-z^{2}} \frac{\partial V}{\partial \varphi} d z,
$$

and $z=\cos \vartheta$. The contour integral in Eq. (8) is taken along the critical energy trajectory or separatrix $\left.\vartheta(\varphi)\right|_{V=V_{0}}$ on which the magnetization may reverse by passing through the saddle point(s) of the energy $v V_{0}$. Equation (6) may be deemed universal in so far as it accurately describes the magnetization escape rate for all damping $\alpha$. Furthermore, the asymptotic behavior of Eq. (7) as a function of $\alpha$, namely, $A\left(\alpha S_{i}\right) \rightarrow 1$ as $\alpha \rightarrow \infty$ and $A\left(\alpha S_{i}\right) / \alpha \rightarrow S_{i}$ as $\alpha \rightarrow 0$, ensures that the IHD and VLD limits of the magnetization escape rate, i.e., Eqs. (3) and (5), respectively, are reproduced correctly. Equation (6) agrees closely with numerical solutions of the Fokker-Planck equation (1), e.g., Refs. 18 and 19, and Langevin dynamics simulations of the magnetization reversal, for example, Refs. 20-22. Equations (3) and (5) have also been successfully compared with experiments ${ }^{23}$ emphasizing the vital importance of an accurate determination of the damping dependence of the escape rate prefactor $A\left(\alpha S_{i}\right) \Omega_{0}(\alpha) \omega_{i} /\left(2 \pi \omega_{0}\right)$ in Eq. (6).

The damping range of validity of presently available asymptotic formulas for magnetization escape rates is summarized in Table I. In practical applications, the conditions of validity of these asymptotic formulas, namely, that they are only valid in the low-temperature (high barrier) limit and for the paraboloid approximation of the free energy in the vicinity of the stationary points, must be taken into account.

\section{REVERSAL TIME FOR MIXED ANISOTROPY}

The salient feature of the IHD equation (3) is the elliptic and hyperbolic paraboloid approximation [Eq. (2)] for the free-energy density $V(\mathbf{M})$ near the relevant stationary points. 

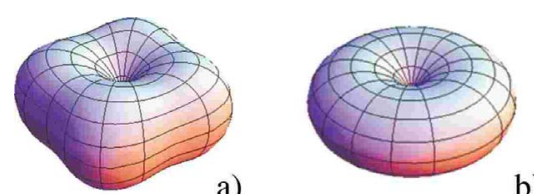

b)

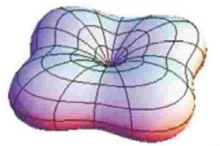

c)

FIG. 1. (Color online) Three-dimensional (3D) plot of the mixed anisotropy potential for $\zeta=1$ (a: flat saddles), 0 (b: uniaxial anisotropy), and -1 (c: flat wells).

However, certain situations exist, where either the well or the damped saddle frequencies or both are zero so that Eq. (3) now predicts zero escape rate. This obviously incorrect result may occur when (i) $V(\mathbf{M})$ is approximately axially symmetric leading to the uniaxial crossover phenomenon, ${ }^{11}$ where the saddle points become simple maxima, or if (ii) the paraboloid approximation [Eq. (2)] fails. The breakdown of the paraboloid approximation (ii) is encountered, for example, for $V(\mathbf{M})$ with either flat saddles or flat well bottoms or both. A particular example is mixed uniaxial and cubic anisotropy

$$
V=K_{1}\left[\sin ^{2} \vartheta+\zeta\left(\sin ^{4} \vartheta \sin ^{2} 2 \varphi+\sin ^{2} 2 \vartheta\right) / 4\right],
$$

where $K_{1}$ denotes the uniaxial anisotropy constant and $\zeta$ is the cubic to uniaxial anisotropy ratio which may be either positive or negative. For $|\zeta| / 4 \gg 1$, potential (9) represents cubic anisotropy, which is a multiwell potential with multiple saddle points.,10 For $|\zeta| \leq 1$, potential (9) represents a double-well potential with two equivalent wells and four equivalent saddle points; these saddle points disappear at $\zeta$ $=0$ corresponding to a uniaxial anisotropy (see Fig. 1). The nearly axially symmetric case (i) was treated in detail by Garanin $\mathrm{et} \mathrm{al.}{ }^{24}$ For $\zeta=-1$ the well frequency $\omega_{i}=0$ while for $\zeta=1$ the damped saddle frequency $\Omega_{0}=0$. These values of $\zeta$ are of particular interest since the existing escape rate formulas described in Sec. I cannot be used to estimate $\tau$ without modification. We remark that the magnetization relaxation rate problem for particles having the mixed anisotropy [Eq. (9)] was implicitly identified by Smith and de Rozario, ${ }^{10}$ Brown, ${ }^{4}$ and Dormann et al. ${ }^{16}$ while Newell ${ }^{25}$ explicitly evaluated the IHD escape rate from Eq. (3), noting the absurd prediction of a vanishing escape rate for $|\zeta|=1$. Nevertheless, the magnetization escape rate problem for nonparaboloidal saddle points and/or wells remains fundamentally unsolved. Now, insofar as the rate calculation for nonparabolic stationary point neighborhoods is concerned, a method for point Brownian particles with separable and additive Hamiltonians has been suggested by Hänggi et $a l .{ }^{26}$ in the IHD limit only. This method has been further generalized ${ }^{27}$ for a Brownian single-axis rotator in a periodic potential with nonparabolic barriers for all damping ranges using the Mel'nikov-Meshkov formalism. ${ }^{6,7}$ Thus our purpose is to extend the escape rate treatment outlined in Refs. 26 and 27 for point particles to a single-domain particle with nonparaboloidal wells/saddles in order to yield a corresponding universal turnover formula for the reversal time of the magnetization $\tau$. We shall confine our treatment to $|\zeta| \leq 1$ for purpose of illustration. The calculations to be described can be extended to $|\zeta|>1$ without major difficulties. For $|\zeta| \gg 1$, where the paraboloid approximations hold, the universal equation (6) has already been checked against numerical calculations of the magnetization relaxation time in Ref. 19.

For mixed anisotropy with $0<|\zeta| \leq 1$, i.e., for a doublewell potential with two equivalent wells and four equivalent saddle points, the reversal time $\tau$ is given in terms of the depopulation factor $A(\delta)$ and $\Gamma_{12}^{\mathrm{IHD}}$ as

$$
\tau=\frac{A(2 \alpha S)}{8 \Gamma_{12}^{\mathrm{IHD}} A^{2}(\alpha S)} .
$$

Here we have noted that $S_{1}=S_{2}=S, \Gamma_{12}^{\mathrm{IHD}}=\Gamma_{21}^{\mathrm{IHD}}$, and the IHD $\Gamma_{12}^{\mathrm{IHD}}$ refers to one saddle point only. The factor 8 occurs because (i) four magnetization escape routes from the well over the saddle points exist and (ii) two equivalent wells are involved in the relaxation process. In order to evaluate the action $S$ in Eq. (10) from contour integral (8), an explicit solution for the separatrix $\left.\vartheta(\varphi)\right|_{V=V_{0}}$ is first required. For the distinct cases of negative and positive cubic anisotropy -1 $\leq \zeta \leq 0$ and $0<\zeta \leq 1$, the respective separatrixes $\left.\vartheta(\varphi)\right|_{V=V_{0}}$ are determined by the trigonometric equations

$$
\begin{gathered}
\sin ^{2} \vartheta+\frac{|\zeta|}{4}\left(\sin ^{4} \vartheta \sin ^{2} 2 \varphi+\sin ^{2} 2 \vartheta\right)=1-\frac{|\zeta|}{4}, \\
\sin ^{2} \vartheta+\frac{\zeta}{4}\left(\sin ^{4} \vartheta \sin ^{2} 2 \varphi+\sin ^{2} 2 \vartheta\right)=1 .
\end{gathered}
$$

The physically meaningful solutions are

$$
\left.\vartheta(\varphi)\right|_{V=V_{0}}=\arccos \sqrt{\frac{|\zeta|(3+\cos 4 \varphi)+4-2 \sqrt{4+8|\zeta|+|\zeta|(|\zeta|-4) \sin ^{2} 2 \varphi}}{|\zeta|(7+\cos 4 \varphi)}}, \quad-1 \leq \zeta \leq 0
$$

and

$$
\left.\vartheta(\varphi)\right|_{V=V_{0}}=\arccos \sqrt{\frac{\zeta(3+\cos 4 \varphi)-4+4 \sqrt{(\zeta-1)^{2}+\zeta \sin ^{2} 2 \varphi}}{\zeta(7+\cos 4 \varphi)}}, \quad 0<\zeta \leq 1 .
$$


By substituting Eqs. (11) and (12) into Eq. (8), we can evaluate the actions $S$ analytically as Taylor-series expansions up to any desired order of $\zeta$ in two distinct regions, viz.,

$$
S=\sigma \sqrt{|\zeta|}\left(1-\frac{7}{12} \zeta-\frac{15}{32} \zeta^{2}-\frac{363}{640} \zeta^{3}-\frac{1569}{2048} \zeta^{4}-\ldots\right)
$$

for negative cubic anisotropy in the range $-0.5 \leq \zeta \leq 0$ and

$$
S=\sigma \sqrt{\zeta}\left(1-\frac{\zeta}{6}+\frac{\zeta^{2}}{8}+\frac{17 \zeta^{3}}{240}-\frac{\zeta^{4}}{128}-\ldots\right)
$$

for positive cubic anisotropy in the entire range of interest. In the remaining negative cubic anisotropy region, $-1 \leq \zeta$ $\leq-0.5, S$ can be computed numerically from Eq. (8).

Thus, the only remaining quantity remaining in Eq. (10) is $\Gamma_{i j}^{\mathrm{IHD}}$, which cannot be evaluated by naively applying Eq. (3) and requires separate analysis for $0 \leq \zeta \leq 1$ and $-1 \leq \zeta<0$. In the first instance, the hyperbolic paraboloid approximation at the saddle point breaks down so that the Kramers method of determining the crossover function between the wells needs to be modified. In the second instance, the elliptic paraboloid approximation at the bottom of the wells breaks down so that the steepest-descent method of calculating the well population needs to be modified. We treat both cases separately as follows.

\section{IHD ESCAPE RATE FOR $0<\zeta \leq 1$}

Despite the breakdown of the hyperbolic paraboloid approximation near the saddle point, the IHD magnetization escape rate can still be expressed as a flux over population. ${ }^{26}$ In order to see this, we first recall that in general in IHD the picture is that inside the well the distribution function of $\mathbf{M}(t)$ is almost the equilibrium Boltzmann distribution. However, very near the saddle the distribution deviates from that equilibrium distribution due to the quasistationary reversal of $\mathbf{M}(t)$ over the saddle point. Now the saddle-point (separatrix) region where nonequilibrium prevails is very small and the saddle point itself is a stationary point; therefore, near that point the Fokker-Planck equation may be written in terms of the direction cosines ${ }^{4}$ of $\mathbf{M}(t)$ as (cf. Sec. V of Ref. 28)

$$
\begin{aligned}
2 \tau_{N} \frac{\partial W}{\partial t} \approx & \frac{\beta}{\alpha}\left(\frac{\partial V}{\partial n_{1}} \frac{\partial W}{\partial n_{2}}-\frac{\partial W}{\partial n_{1}} \frac{\partial V}{\partial n_{2}}\right)+\frac{\partial}{\partial n_{1}}\left(\beta W \frac{\partial V}{\partial n_{1}}+\frac{\partial W}{\partial n_{1}}\right) \\
& +\frac{\partial}{\partial n_{2}}\left(\beta W \frac{\partial V}{\partial n_{2}}+\frac{\partial W}{\partial n_{2}}\right) .
\end{aligned}
$$

Since the barrier crossing process is exponentially slow, we may now assume a quasistationary solution of Eq. (15) in the separatrix region of the form

$$
W\left(n_{1}, n_{2}, t\right)=w\left(n_{1}, n_{2}\right) e^{-\Gamma_{i j}^{\mathrm{IHD}} t},
$$

leading to

$$
\begin{aligned}
-2 \Gamma_{i j}^{\mathrm{IHD}} \tau_{N} w \approx & \frac{\beta}{\alpha}\left(\frac{\partial V}{\partial n_{1}} \frac{\partial w}{\partial n_{2}}-\frac{\partial w}{\partial n_{1}} \frac{\partial V}{\partial n_{2}}\right) \\
& +\frac{\partial}{\partial n_{1}}\left(\beta w \frac{\partial V}{\partial n_{1}}+\frac{\partial w}{\partial n_{1}}\right) \\
& +\frac{\partial}{\partial n_{2}}\left(\beta w \frac{\partial V}{\partial n_{2}}+\frac{\partial w}{\partial n_{2}}\right) .
\end{aligned}
$$

Thus by integrating this equation with respect to the direction cosines $n_{1}$ and $n_{2}$, limiting the integration to a single well, then using Green's theorem in the $\left(n_{1}, n_{2}\right)$ plane, we may formally obtain (see Appendix A) $\Gamma_{i j}^{\mathrm{IHD}}$ as the closed line integral along the saddle-point contour

$$
\begin{aligned}
-2 \alpha \Gamma_{i j}^{\mathrm{IHD}} \tau_{N} \approx & Z_{i}^{-1} \oint_{\text {well } i \text { boundary }} e^{-\beta V}\left\{\left(\beta g \frac{\partial V}{\partial n_{2}}-\alpha \frac{\partial g}{\partial n_{1}}\right) d n_{2}\right. \\
& \left.+\left(\beta g \frac{\partial V}{\partial n_{1}}+\alpha \frac{\partial g}{\partial n_{2}}\right) d n_{1}\right\} .
\end{aligned}
$$

Here $g=e^{\beta V} w$ is the crossover function ${ }^{11,24}$ originally used by Kramers $^{5}$ to obtain the IHD solution of the Klein-Kramers equation pertaining to point particles by converting that equation into an ordinary differential equation. The partition function $Z_{i} \approx \iint_{\text {well } i} W\left(n_{1}, n_{2}\right) d n_{1} d n_{2}$ represents the well population, where of course the elliptic paraboloid approximation for the energy near the bottom of the well still holds so that $Z_{i}$ may be evaluated as usual by steepest descents. Estimation of the saddle-point contour integral in Eq. (18) for $V$ given by Eq. (9) with $0 \leq \zeta \leq 1$ (Appendix A) then yields

$$
\begin{aligned}
\tau_{0} \Gamma_{i j}^{\mathrm{IHD}} \sim & \sqrt{1+\frac{\zeta \sigma}{A_{1}}} \frac{\sigma(1+\zeta)(1-\alpha q) K_{1 / 4}\left[\left(A_{1}+\zeta \sigma\right)^{2} /\left(8 A_{2}\right)\right]}{2 \pi\left(1+\alpha^{2}\right) K_{1 / 4}\left[A_{1}^{2} /\left(8 A_{2}\right)\right]} \\
& \times e^{\zeta \sigma\left(\zeta \sigma+2 A_{1}\right) / 8 A_{2}-\sigma},
\end{aligned}
$$

where $\sigma=\beta K_{1}$,

$$
\begin{gathered}
q=-(2 \zeta)^{-1}\left[\alpha+\sqrt{\alpha^{2}+4 \zeta(1-\zeta)}\right], \\
A_{1}=-\frac{\zeta \sigma(q+\alpha)}{\alpha\left(1+q^{2}\right)}, \quad A_{2}=-\frac{(\alpha q-1) \zeta \sigma}{\alpha q^{3}\left(1+q^{2}\right)},
\end{gathered}
$$

and $K_{1 / 4}(z)$ is a modified Bessel function of the third kind. ${ }^{29}$ On further noting that for $x \rightarrow+0,{ }^{29}$

$$
e^{x} K_{1 / 4}(x) \approx \Gamma(1 / 4)(x / 2)^{-1 / 4} / 2+\ldots,
$$

where $\Gamma(z)$ is the gamma function, ${ }^{29} \mathrm{Eq}$. (19) becomes for $\zeta=1$

$$
\tau_{0} \Gamma_{i j}^{\mathrm{IHD}} \sim \frac{\alpha \sigma^{5 / 4}}{\pi \Gamma(1 / 4)} K_{1 / 4}\left(\alpha^{4} \sigma / 8\right) e^{-\sigma\left(1-\alpha^{4} / 8\right)} .
$$

For $\alpha^{4} \sigma / 8 \gg 1$, noting that $e^{x} K_{1 / 4}(x) \sim(\pi / 2 x)^{1 / 2}$ at $x \rightarrow \infty,{ }^{29}$ we have from Eq. (23)

$$
\tau_{0} \Gamma_{i j}^{\mathrm{IHD}} \sim \frac{2 \sigma^{3 / 4} e^{-\sigma}}{\alpha \sqrt{\pi} \Gamma(1 / 4)},
$$

which combined with Eq. (10) completes the solution of the flat saddle problem. 


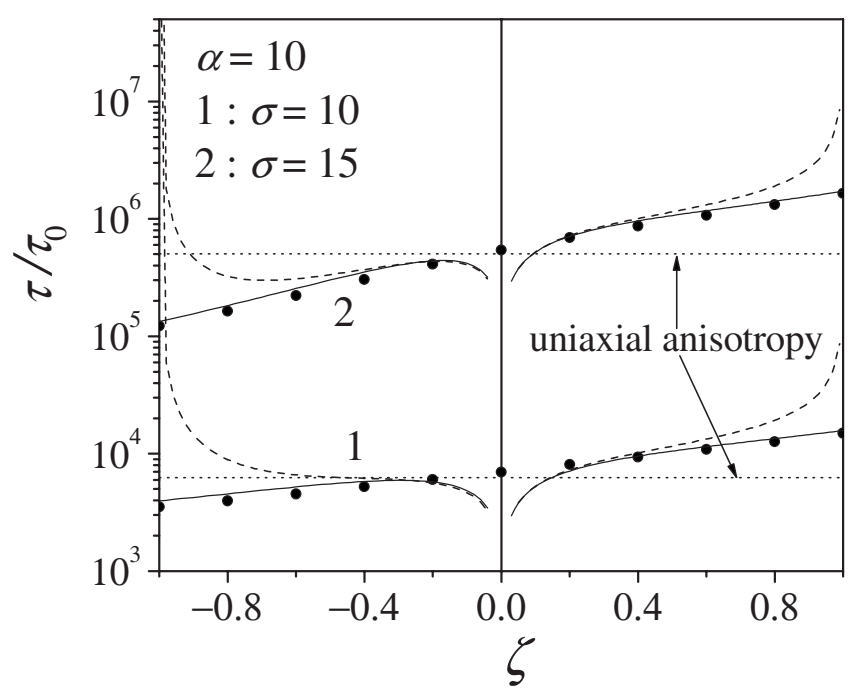

FIG. 2. The normalized reversal time of the magnetization $\tau / \tau_{0}$ as a function of the potential shape parameter $\zeta$ for $\alpha=10$ and $\sigma$ $=10$ and 15. Filled circles: numerical calculation from Eq. (32), solid lines: universal Mel'nikov-Meshkov formula (10), dashed lines: Eq. (33), dotted lines: Eq. (35).

\section{IHD ESCAPE RATE FOR $-1 \leq \zeta \leq 0$}

We now consider negative anisotropy ratio, $-1 \leq \zeta \leq 0$, where free-energy potential (8) near the bottom of a well may not be approximated by an elliptic paraboloid, which will obviously affect the well partition function. Nevertheless $\Gamma_{i j}^{\mathrm{IHD}}$ can still be estimated from Langer's expression, viz., ${ }^{12,26}$

$$
\Gamma_{i j}^{\mathrm{IHD}}=\frac{\Omega_{0}}{2 \pi} \frac{Z_{0}}{Z_{i}}
$$

where $\quad Z_{i} \approx \iint_{\text {well }} e^{-\beta V(\vartheta, \varphi)} \sin \vartheta d \vartheta d \varphi$ and $Z_{0}$ $\approx \iint_{\text {saddle }} e^{-\beta V(\vartheta, \varphi)} \sin \vartheta d \vartheta d \varphi$ are the well and saddle partition functions, respectively. First, we recall that near the saddle for $-1 \leq \zeta<0$, the hyperbolic paraboloid approximation still holds so that $Z_{0}$ and the damped saddle frequency $\Omega_{0}$ can be evaluated as usual. Hence,

$$
Z_{0}=2 \pi[|\zeta|(2+|\zeta|)]^{-1 / 2} e^{-\sigma(1-|\zeta| / 4)}
$$

and

$$
\Omega_{0}=\frac{\sigma}{4 \tau_{0}\left(\alpha+\alpha^{-1}\right)}\left[2-|\zeta|+\sqrt{(3|\zeta|+2)^{2}+8 \alpha^{-2}|\zeta|(2+|\zeta|)}\right] .
$$

Thus we only have to calculate the well partition function $Z_{i}$, where the elliptic paraboloid approximation fails. $Z_{i}$ may now be evaluated using a method detailed in Appendix B yielding

$$
Z_{i} \approx \frac{(1-|\zeta|)}{4|\zeta|} e^{\sigma(1-|\zeta|)^{2} / 4|\zeta|} K_{1 / 4}^{2}\left[\frac{\sigma(1-|\zeta|)^{2}}{8|\zeta|}\right] .
$$

Thus by substituting Eqs. (26)-(28) into Eq. (25), we finally obtain

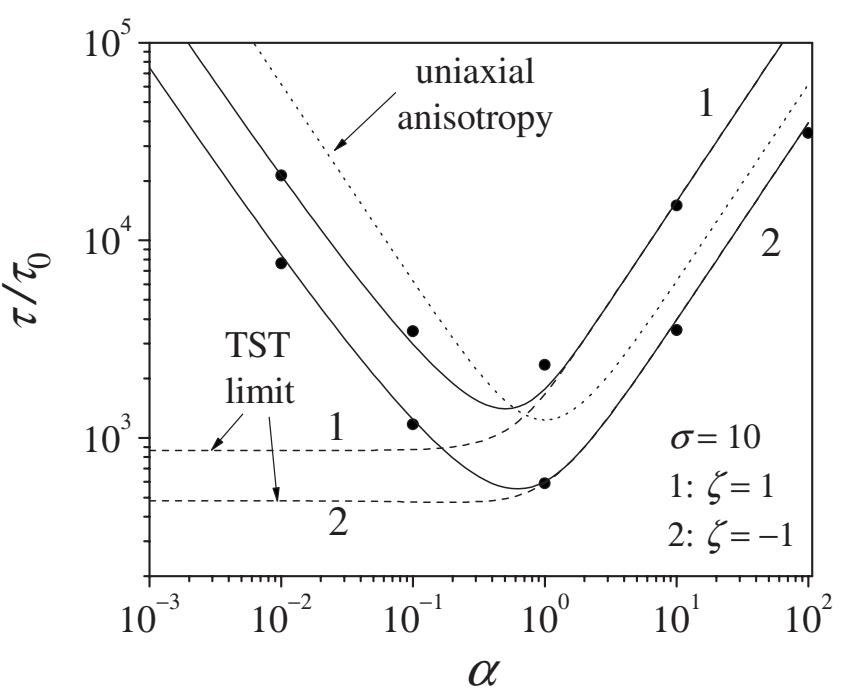

FIG. 3. The normalized reversal time of the magnetization $\tau / \tau_{0}$ as a function of the damping parameter $\alpha$ for $\zeta=-1$ and $\zeta=1$. Filled circles: numerical calculation from Eq. (32). Solid lines: universal Mel'nikov-Meshkov formula (10). Dashed lines: Eqs. (30) and (23). Dotted line: uniaxial Eq. (35). The predictions of TST, viz., $\tau$ $\sim\left(8 \Gamma_{i j}^{\mathrm{TST}}\right)^{-1}$, are also shown for comparison.

$$
\begin{aligned}
\tau_{0} \Gamma_{i j}^{\mathrm{IHD}} & \\
& \sim \sqrt{\frac{|\zeta|}{2(2+|\zeta|)}} \frac{\left[2-|\zeta|+\sqrt{(2+3|\zeta|)^{2}+8 \alpha^{-2}|\zeta|(2+|\zeta|)}\right]}{\left(\alpha+\alpha^{-1}\right)(1-|\zeta|) K_{1 / 4}^{2}\left[(1-|\zeta|)^{2} \sigma /(8|\zeta|)\right]} \\
& \times e^{-\sigma(1+2|\zeta|) /(4|\zeta|)} .
\end{aligned}
$$

On noting Eq. (22), Eq. (29) for $\zeta=-1$ becomes

$$
\tau_{0} \Gamma_{i j}^{\mathrm{IHD}} \sim \frac{\sqrt{\sigma / 6}\left(\alpha+\sqrt{25 \alpha^{2}+24}\right)}{\left(1+\alpha^{2}\right) \Gamma^{2}(1 / 4)} e^{-3 \sigma / 4} .
$$

This equation, combined with Eq. (10), completes the flat minimum magnetization escape rate.

\section{RESULTS AND DISCUSSION}

In order to verify the universal escape rate [Eq. (10)] as modified for mixed anisotropy, we shall use the matrix continued fraction method as developed in Refs. 30 and 31 for the solution of the Fokker-Planck equation (1) for cubic anisotropy. By applying this method to mixed anisotropy [Eq. (9)], the solution of Eq. (1) reduces to solving an infinite hierarchy of differential-recurrence relations for the equilibrium correlation functions $c_{l, m}(t)$ $=\left\langle\cos \vartheta(0) Y_{l, m}[\vartheta(t), \varphi(t)]\right\rangle_{0}$,

$$
\frac{d}{d t} c_{l, m}(t)=\sum_{l^{\prime}, m^{\prime}} d_{l^{\prime}, m^{\prime}, l, m} c_{l^{\prime}, m^{\prime}}(t),
$$

where $Y_{l, m}(\vartheta, \varphi)$ is a normalized spherical harmonic and $d_{l^{\prime}, m^{\prime}, l, m}$ are the matrix elements of the Fokker-Planck operator in Eq. (1). The derivation of the moment system [Eq. (31)] for an arbitrary free energy is given in Ref. 32 (see also Ref. 33, Chap. 7). The differential-recurrence relation [Eq. (31)] which has 27 recurring terms can be solved by matrix 
continued fractions. Details of this algorithm allowing us to calculate the longitudinal correlation time, viz.,

$$
\tau_{\|}=\frac{1}{c_{1,0}(0)} \int_{0}^{\infty} c_{1,0}(t) d t
$$

can be found elsewhere. ${ }^{33}$ For mixed anisotropy with $|\zeta| \leq 1$, we have $\tau_{\|} \approx \tau$, thus providing a useful numerical check of Eq. (10).

The results of the numerical matrix continued fraction and asymptotic [from Eqs. (10), (19), (23), (29), and (30)] calcu- lations of the normalized reversal time $\tau / \tau_{0}$ as functions of the anisotropy ratio parameter $\zeta$ and damping parameter $\alpha$ are shown in Figs. 2 and 3, respectively. Figure 3 shows that the universal equation (10) describes the behavior of the reversal time in the entire dissipation range for both $\zeta=-1$ and $\zeta=1$. Moreover, Eq. (10) is valid in the range $0.2 \leq|\zeta| \leq 1$ (see Fig. 2, where the results presented are for a particular IHD value of $\alpha$ ). Here, the usual IHD formula [Eq. (3)] based on the paraboloidal approximation does not describe the relaxation rate at all as is apparent from Fig. 2. In fact, for mixed anisotropy, Eq. (3) yields

$$
\Gamma_{i j}^{\mathrm{IHD}} \approx \begin{cases}\frac{\sigma(1+\zeta)\left[1-2 \zeta+\sqrt{1+4 \alpha^{-2} \zeta(1-\zeta)}\right] e^{-\sigma}}{4 \pi \tau_{0}\left(\alpha+\alpha^{-1}\right) \sqrt{\zeta(1-\zeta)}}, & 0 \leq \zeta \leq 1, \\ \frac{\sigma(1-|\zeta|)\left[2-|\zeta|+\sqrt{(2+3|\zeta|)^{2}+8 \alpha^{-2}|\zeta|(2+|\zeta|)}\right] e^{-\sigma(1-|\zeta| / 4)}}{4 \pi \tau_{0}\left(\alpha+\alpha^{-1}\right) \sqrt{2|\zeta|(2+|\zeta|)}}, & -1 \leq \zeta \leq 0 .\end{cases}
$$

By inspection, Eq. (33) predicts zero escape rate for $|\zeta|=1$, while yielding an infinite escape rate as $|\zeta| \rightarrow 0$. Figure 2 also indicates that Eqs. (10), (19), (23), (29), and (30) reproduce correctly the behavior of the relaxation time for $0.2 \leq|\xi|$ $\leq 1$ in contrast to Eq. (3). Notice that the correct escape rate equation for the uniaxial case, $\zeta=0$, is in fact provided by Brown's uniaxial anisotropy formula ${ }^{3}$

$$
\Gamma_{12}=\Gamma_{21}=\frac{\sigma^{3 / 2} e^{-\sigma}}{\tau_{0}\left(\alpha+\alpha^{-1}\right) \sqrt{\pi}},
$$

which allows one to estimate $\tau^{-1}$ as

$$
\tau^{-1}=2 \Gamma_{12} \text {. }
$$

Equation (35) is valid for all values of the dissipation parameter $\alpha$. The reversal time given by Eq. (34) agrees in all respects with the relaxation time rendered by Eq. (32) for $\zeta$ $=0$ provided that the barrier height $\sigma$ is large, as expected from the calculation of the correlation time of the magnetization in uniaxial potentials (see Fig. 2). In this context, we remark that as $|\zeta| \rightarrow 0$, axial symmetry is attained and the azimuthal dependence of the distribution function disappears. Thus for arbitrary damping, Eq. (1) becomes a onedimensional Fokker-Planck equation for the distribution function $W(\vartheta)$, which due to axial symmetry is independent of the azimuthal angle $\varphi$, viz., ${ }^{3}$

$$
2 \tau_{N} \frac{\partial W}{\partial t}=\frac{1}{\sin \vartheta} \frac{\partial}{\partial \vartheta}\left[\sin \vartheta\left(\frac{\partial W}{\partial \vartheta}+\beta W \frac{\partial V}{\partial \vartheta}\right)\right] .
$$

The saddle region now becomes infinitely wide so that the method of Garanin et al. ${ }^{24}$ should be applied as the Mel'nikov-Meshkov method fails since the action $S$ is zero in this case, once again yielding zero escape rate. By comparing the uniaxial asymptotes Eqs. (34) and (35) with Eq. (10) (see Fig. 3), we see that $\tau$ for pure uniaxial anisotropy can differ by as much as an order of magnitude from $\tau$ for mixed anisotropy as rendered by Eq. (10). This fact may be attributed to the difference in the prefactors between the uniaxial and nonaxially symmetric results. In particular, for $\zeta=1$ and $\alpha \ll 1$, Eq. (23) predicts the same barrier height as Eq. (34); however, the particular dependence of the prefactor on the damping $\alpha$ and barrier height parameter $\sigma$ renders a different reversal time.

In conclusion, we have obtained accurate formulas for the reversal time of the magnetization $\tau$ of single-domain ferromagnetic particles with mixed uniaxial and cubic anisotropy valid for all values of damping. This anisotropy is characterized by an energy landscape with nonparaboloidal saddles and/or well bottoms in certain ranges of cubic to uniaxial anisotropy constant ratios. The method may be extended to other potentials for which the second-order Taylor expansion coefficients of the potential equation (2) vanish at the relevant stationary points. We remark that the mixed anisotropy [Eq. (9)] appears in various applications. Note the "effective macrospin" model ${ }^{34,35}$ whereby a many-spin cluster is mapped onto a macrospin representing the net moment of the cluster with the corresponding energy comprising mixed uniaxial and cubic anisotropies. Here the effective anisotropy energy landscape depends on the size and shape of the cluster, the crystalline structure of the underlying material, and the other physical parameters such as the exchange coupling and local anisotropy constants. The model provides a compromise between the macrospin approach based on the Stoner-Wohlfarth concept ${ }^{36}$ of coherent rotation of all the atomic spins and the many-spin approach. ${ }^{37}$ The effective constants of the model, e.g., the parameter $\zeta$, etc., must, however, be computed from microscopic considerations in order to account for the crystallographic structure, the shape of the particle, the number of spins, etc. so that the model can be directly compared with experimental data or numerical simulations. ${ }^{38}$ Furthermore, our results may also be applied 
to describe the temperature dependence of the switching field curves of isolated Co nanoclusters characterized by mixed anisotropy. ${ }^{39}$ In Ref. 40 the temperature-dependent switching field curves of such a magnetic nanocluster having mixed anisotropy (9) were calculated; and, in particular, it was shown that temperature-dependent switching field curves noticeably deviate from the Stoner-Wohlfarth astroid for uniaxial particles. Mixed anisotropy energy (9) also occurs in paleomagnetism and rock magnetism, ${ }^{25}$ where thermal relaxation is important for both thermoremanent magnetization and related measurements determining the blocking temperature(s) characteristic of a given material. Here the anisotropy energy (9) permits many remanent states to coexist for $|\zeta|>1$, thereby leading to multiple blocking temperatures and a transition toward a single blocking temperature. ${ }^{25}$

\section{ACKNOWLEDGMENT}

The authors are grateful to H. Kachkachi and L. Cleary for helpful discussions.

\section{APPENDIX A: DERIVATION OF EQ. (19)}

In order to evaluate the escape rate from Eq. (18), an expression for the relevant Kramers crossover function $g$ $=e^{\beta V} w$ and its first derivatives at the well boundary along with a suitable parametrization of the well boundary itself is required. This can be accomplished as follows. Since the distribution function $w$ must always be finite and because a large barrier is assumed, the left-hand side of Eq. (17) almost vanishes by quasistationarity; hence, in terms of the Kramers crossover function $g$ we have ${ }^{28}$

$$
\left[\frac{\partial V}{\partial n_{2}}+\alpha \frac{\partial V}{\partial n_{1}}\right] \frac{\partial g}{\partial n_{1}}-\left[\frac{\partial V}{\partial n_{1}}-\alpha \frac{\partial V}{\partial n_{2}}\right] \frac{\partial g}{\partial n_{2}} \approx \frac{\alpha}{\beta}\left[\frac{\partial^{2} g}{\partial n_{1}^{2}}+\frac{\partial^{2} g}{\partial n_{2}^{2}}\right] .
$$

Next, by approximating $V$ near a saddle point to fourth order in the direction cosines $\left(n_{1}, n_{2}\right)$, we have $V$ as

$$
\beta V\left(n_{1}, n_{2}\right) \approx \sigma-\sigma(1-\zeta) n_{1}^{2}+\zeta \sigma n_{2}^{2}-\zeta \sigma\left(n_{1}^{4}+n_{1}^{2} n_{2}^{2}+n_{2}^{4}\right) .
$$

Following Hänggi et al., ${ }^{26}$ we may now seek $g$ as

$$
g\left(n_{1}, n_{2}\right)=C^{-1} \int_{-\infty}^{\rho\left(n_{1}, n_{2}\right)} e^{-A_{1} z^{2}-A_{2} z^{4}} d z
$$

where $C=\int_{-\infty}^{+\infty} e^{-A_{1} z^{2}-A_{2} z^{4}} d z$ is a normalization constant such that $g(\rho=+\infty)=1$, where $A_{1}$ and $A_{2}$ are unknown coefficients accounting for both the shape of the saddle region and the energy loss at the saddle, and where $\rho\left(n_{1}, n_{2}\right)$ is a function to be determined (this function measures the "distance" from the saddle-point location). We note in passing that the Kramers IHD calculation as adapted to magnetization reversal by Brown $^{3,4}$ corresponds to setting $A_{2}=0$ in Eq. (A3) and dropping the fourth-order terms in the Taylor-series expansion of the free-energy density in Eq. (A2) altogether. Here in contrast, we must have $A_{2} \neq 0$ in order to account for the nonparaboloidal shape of the saddle, and all terms of the fourth- order Taylor expansion of the free-energy density in Eq. (A2) are retained in Eq. (A1), et seq. In succinct terms, because of the nonparaboloidal shape of the saddle region, the crossover function deviates from the error function originally used by Kramers for parabolic barriers.

Next one must transform the partial differential Eq. (A1) in the two variables $\left(n_{1}, n_{2}\right)$ into an ordinary linear differential equation in the single variable $\rho$. We may do this following Kramers ${ }^{5}$ by implicitly seeking $\rho$ as a linear combination of $n_{1}$ and $n_{2}$ in the saddle region. The details of the procedure are as follows. By substituting crossover function (A3) into Eq. (A1), we obtain a nonlinear partial differential equation for $\rho\left(n_{1}, n_{2}\right)$, viz.,

$$
\begin{aligned}
{\left[\frac{\partial V}{\partial n_{2}}+\alpha \frac{\partial V}{\partial n_{1}}\right] \frac{\partial \rho}{\partial n_{1}}-\left[\frac{\partial V}{\partial n_{1}}-\alpha \frac{\partial V}{\partial n_{2}}\right] \frac{\partial \rho}{\partial n_{2}} } \\
\approx-\frac{\alpha}{\beta}\left(2 A_{1} \rho+4 A_{2} \rho^{3}\right)\left[\left(\frac{\partial \rho}{\partial n_{1}}\right)^{2}+\left(\frac{\partial \rho}{\partial n_{2}}\right)^{2}\right] \\
+\frac{\alpha}{\beta}\left[\frac{\partial^{2} \rho}{\partial n_{1}^{2}}+\frac{\partial^{2} \rho}{\partial n_{2}^{2}}\right] .
\end{aligned}
$$

Now, we seek the conditions for which Eq. (A4) can be reduced to an ordinary differential equation for $\rho$. By introducing, again following Kramers, ${ }^{5}$ a new variable in the form of the linear combination $r=q n_{1}+n_{2}$ in Eq. (A4), we have

$$
\begin{aligned}
{\left[(q+\alpha) \frac{\partial V}{\partial n_{2}}+(\alpha q-1) \frac{\partial V}{\partial n_{1}}\right] \frac{d \rho}{d r} } \\
=-\frac{\alpha}{\beta}\left(1+q^{2}\right)\left(2 A_{1} \rho+4 A_{2} \rho^{3}\right)\left(\frac{d \rho}{d r}\right)^{2} \\
+\frac{\alpha}{\beta}\left(1+q^{2}\right) \frac{d^{2} \rho}{d r^{2}},
\end{aligned}
$$

where we reiterate that all the terms of the Taylor-series expansion in Eq. (A2) should be included when computing the derivatives $\partial V / \partial n_{i}$, unlike the Kramers-Langer-Brown calculation, where the fourth-order terms are dropped. Because $\rho$ is implicitly sought as a linear combination of $n_{1}$ and $n_{2}$, it follows that $\rho$ and $r$ are proportional to each other so that the second-order derivative $d^{2} \rho / d r^{2}$ is zero. Hence, the above equation becomes

$$
\begin{aligned}
(q+ & \alpha)\left(\zeta \sigma n_{2}-2 \zeta \sigma n_{1}^{2} n_{2}-4 \zeta \sigma n_{2}^{3}\right)+(1-\alpha q)\left[\sigma(1-\zeta) n_{1}\right. \\
& \left.+2 \zeta \sigma n_{1} n_{2}^{2}+4 \zeta \sigma n_{1}^{3}\right]=-\frac{\alpha}{\beta}\left(1+q^{2}\right)\left(2 A_{1} \rho+4 A_{2} \rho^{3}\right) \frac{d \rho}{d r}
\end{aligned}
$$

where we have used Eq. (A2). Next, expressing $n_{1}$ as $(r$ $\left.-n_{2}\right) / q$ in the left-hand side of Eq. (A5) and expanding the powers of $r-n_{2}$, we may choose $q$ so that the terms linear in $n_{2}$ cancel and then set $n_{2}=0$ in the remaining terms. The quadratic equation determining $q$ is

$$
q(q+\alpha) \zeta=(\alpha q-1)(1-\zeta) .
$$

Since this equation has two real roots (one positive and one negative), one must choose the root permitting normalization of the crossover function $g$, i.e., $q$ as rendered by Eq. (20), 
which represents the unstable barrier crossing mode. Now, Eq. (A5) becomes the proper first-order nonlinear ordinary differential equation

$$
\begin{aligned}
2(q & +\alpha) \zeta \sigma r+4(\alpha q-1) \zeta \sigma q^{-3} r^{3} \\
& =-2 \alpha\left(1+q^{2}\right)\left(A_{1} \rho+2 A_{2} \rho^{3}\right) \frac{d \rho}{d r} .
\end{aligned}
$$

On integrating this equation with respect to $r$ with boundary condition $\rho(r=0)=0$, we have

$$
(q+\alpha) \zeta \sigma r^{2}+(\alpha q-1) \zeta \sigma q^{-3} r^{4}=-\alpha\left(1+q^{2}\right)\left(A_{1} \rho^{2}+A_{2} \rho^{4}\right) .
$$

This last equation is a biquadratic equation for $\rho$, the physically meaningful solution of which is $\rho=r$, provided that the coefficients $A_{1}$ and $A_{2}$ are given by Eq. (21). These expressions for $\rho, A_{1}$, and $A_{2}$ completely determine the parameters of the Kramers crossover function $g$.

Having determined $g$, we may now evaluate the escape rate from Eq. (18). The well partition function $Z_{i}$ can be estimated by steepest descents. For $V$ given by Eq. (9), we obtain

$$
Z_{i}=\int_{0}^{2 \pi} \int_{0}^{\pi / 2} e^{-\beta V(\vartheta, \varphi)} \sin \vartheta d \vartheta d \varphi \approx \frac{\pi}{\sigma(1+\zeta)} .
$$

According to Brown ${ }^{4}$ and Geoghegan et al., ${ }^{28}$ the well boundary is parametrized by $n_{1}=0$, therefore putting $d n_{1}=0$ in the contour integral [Eq. (18)]; and retaining the parabolic approximation only in the factor $e^{-\beta V\left(0, n_{2}\right)}$, Eq. (18) becomes finally

$$
\Gamma_{i j}^{\mathrm{IHD}} \approx \frac{\sigma(1+\zeta)(1-\alpha q)}{2 \pi \tau_{0}\left(1+\alpha^{2}\right)} C^{-1} \int_{-\infty}^{+\infty} e^{-\left(\zeta \sigma+A_{1}\right) n_{2}^{2}-A_{2} n_{2}^{4}} d n_{2},
$$

which on account of the identity ${ }^{41}$

$$
\int_{0}^{\infty} e^{-a z^{2}-b z^{4}} d z=\frac{1}{4} \sqrt{\frac{a}{b}} e^{a^{2} /(8 b)} K_{1 / 4}\left(\frac{a^{2}}{8 b}\right)
$$

yields the flat saddle escape rate (19).

\section{APPENDIX B: WELL PARTITION FUNCTION FOR $-\mathbf{1} \leq \zeta<0$}

Here we derive Eq. (28) for the partition function $Z_{i}$ of a well $i$ with an almost flat minimum. First, we note that $Z_{i}$ may be approximated in the low-temperature limit as

$$
\begin{aligned}
Z_{i} & =\int_{0}^{2 \pi} \int_{0}^{\pi / 2} e^{-\beta V(\vartheta, \varphi)} \sin \vartheta d \vartheta d \varphi \\
& \approx \int_{-\infty}^{\infty} \int_{-\infty}^{\infty} e^{-\beta V\left(n_{1}, n_{2}\right)} d n_{1} d n_{2} .
\end{aligned}
$$

By expanding $\beta V$ near a minimum, say $(\vartheta=0, \varphi=0)$ in terms of the direction cosines of the magnetization $\left(n_{1}, n_{2}, n_{3}\right)$ and using $n_{3}=\sqrt{1-n_{1}^{2}-n_{2}^{2}} \approx 1-2^{-1}\left(n_{1}^{2}+n_{2}^{2}\right)-8^{-1}\left(n_{1}^{2}+n_{2}^{2}\right)^{2}$, we obtain the following Taylor expansion:

$$
\begin{aligned}
\beta V(\vartheta, \varphi) & \rightarrow \beta V\left(n_{1}, n_{2}\right) \\
& \approx \sigma(1-|\zeta|)\left(n_{1}^{2}+n_{2}^{2}\right)+\sigma|\zeta|\left(n_{1}^{4}+n_{1}^{2} n_{2}^{2}+n_{2}^{4}\right) .
\end{aligned}
$$

Now, on using Eq. (B2) and identity (A8), Eq. (B1) reduces to

$$
\begin{aligned}
Z_{i} \approx & 2 \int_{-\infty}^{+\infty} \sqrt{\frac{1-|\zeta|+|\zeta| n_{2}^{2}}{16|\zeta|}} e^{\sigma\left(1-|\zeta|+|\zeta| n_{2}^{2}\right)^{2} / 8|\zeta|} \\
& \times K_{1 / 4}\left[\frac{\sigma\left(1-|\zeta|+|\zeta| n_{2}^{2}\right)^{2}}{8|\zeta|}\right] e^{-\sigma\left[(1-|\zeta|) n_{2}^{2}-|\zeta| n_{2}^{4}\right]} d n_{2} .
\end{aligned}
$$

Noting that the integrand has a maximum near $n_{2} \approx 0$, Eq. (B3) can be further simplified to yield Eq. (28), which approximates the well partition function $Z_{i}$ with an error on the order of $5 \%$ for $|\zeta| \leq 1$.
${ }^{1}$ L. Néel, Ann. Geophys. (C.N.R.S.) 5, 99 (1949).

${ }^{2}$ C. P. Bean and J. D. Livingston, J. Appl. Phys. 30, S120 (1959).

${ }^{3}$ W. F. Brown, Jr., Phys. Rev. 130, 1677 (1963).

${ }^{4}$ W. F. Brown, Jr., IEEE Trans. Magn. 15, 1196 (1979).

${ }^{5}$ H. A. Kramers, Physica 7, 284 (1940).

${ }^{6}$ V. I. Mel'nikov and S. V. Meshkov, J. Chem. Phys. 85, 1018 (1986).

${ }^{7}$ V. I. Mel'nikov, Phys. Rep. 209, 1 (1991).

${ }^{8}$ H. Grabert, Phys. Rev. Lett. 61, 1683 (1988).

${ }^{9}$ E. Pollak, H. Grabert, and P. Hänggi, J. Chem. Phys. 91, 4073 (1989).

${ }^{10}$ D. A. Smith and F. A. de Rozario, J. Magn. Magn. Mater. 3, 219 (1976).

${ }^{11}$ W. T. Coffey, D. A. Garanin, and D. J. McCarthy, Adv. Chem. Phys. 117, 528 (2001).

${ }^{12}$ J. S. Langer, Ann. Phys. (N.Y.) 54, 258 (1969).
${ }^{13}$ R. Becker and W. Döring, Ann. Phys. 416, 719 (1935).

${ }^{14}$ I. Klik and L. Gunther, J. Appl. Phys. 67, 4505 (1990).

${ }^{15}$ I. Klik and L. Gunther, J. Stat. Phys. 60, 473 (1990).

${ }^{16}$ J. L. Dormann, D. Fiorani, and E. Tronc, Adv. Chem. Phys. 98, 283 (1997).

${ }^{17}$ P. M. Déjardin, D. S. F. Crothers, W. T. Coffey, and D. J. McCarthy, Phys. Rev. E 63, 021102 (2001).

${ }^{18}$ Yu. P. Kalmykov, J. Appl. Phys. 96, 1138 (2004); 101, 093909 (2007).

${ }^{19}$ Yu. P. Kalmykov, W. T. Coffey, B. Ouari, and S. V. Titov, J. Magn. Magn. Mater. 292, 372 (2005); Yu. P. Kalmykov, W. T. Coffey, and S. V. Titov, Fiz. Tverd. Tela (St. Petersburg) 47, 260 (2005) [Phys. Solid. State 47, 272 (2005)]; Yu. P. Kalmykov and B. Ouari, Phys. Rev. B 71, 094410 (2005); B. Ouari and Yu. P. Kalmykov, J. Appl. Phys. 100, 123912 (2006).

${ }^{20} \mathrm{C}$. Vouille, A. Thiaville, and J. Miltat, J. Magn. Magn. Mater. 
272-276, E1237 (2004).

${ }^{21}$ X. Z. Cheng, M. B. A. Jalil, H. K. Lee, and Y. Okabe, J. Appl. Phys 99, 08B901 (2006).

${ }^{22}$ H. J. Suh, C. Heo, C. Y. You, W. Kim, T. D. Lee, and K. J. Lee, Phys. Rev. B 78, 064430 (2008).

${ }^{23}$ W. T. Coffey, D. S. F. Crothers, J. L. Dormann, Yu. P. Kalmykov, E. C. Kennedy, and W. Wernsdorfer, Phys. Rev. Lett. 80, 5655 (1998).

${ }^{24}$ D. A. Garanin, E. C. Kennedy, D. S. F. Crothers, and W. T. Coffey, Phys. Rev. E 60, 6499 (1999).

${ }^{25}$ A. J. Newell, Geochem., Geophys., Geosyst. 7, Q03015 (2006); 7, Q03016 (2006).

${ }^{26}$ P. Hänggi, P. Talkner, and M. Borkovec, Rev. Mod. Phys. 62, 251 (1990).

${ }^{27}$ R. Bastardis, P. M. Déjardin, and Yu. P. Kalmykov, Physica A 387, 3432 (2008).

${ }^{28}$ L. J. Geoghegan, W. T. Coffey, and B. Mulligan, Adv. Chem. Phys. 100, 475 (1997)

${ }^{29}$ Handbook of Mathematical Functions, edited by M. Abramowitz and I. Stegun (Dover, New York, 1964).

${ }^{30}$ Yu. P. Kalmykov and S. V. Titov, Zh. Eksp. Teor. Fiz. 115, 101 (1999) [JETP 88, 58 (1999)].

${ }^{31}$ Yu. P. Kalmykov, S. V. Titov, and W. T. Coffey, Phys. Rev. B
58, 3267 (1998); Yu. P. Kalmykov, ibid. 61, 6205 (2000).

${ }^{32}$ Yu. P. Kalmykov and S. V. Titov, Phys. Rev. Lett. 82, 2967 (1999); J. Magn. Magn. Mater. 210, 233 (2000).

${ }^{33}$ W. T. Coffey, Yu. P. Kalmykov, and J. T. Waldron, The Langevin Equation, 2nd ed. (World Scientific, Singapore, 2004).

${ }^{34}$ D. A. Garanin and H. Kachkachi, Phys. Rev. Lett. 90, 065504 (2003).

${ }^{35}$ H. Kachkachi and E. Bonet, Phys. Rev. B 73, 224402 (2006).

${ }^{36}$ E. C. Stoner and E. P. Wohlfarth, Philos. Trans. R. Soc. London. Ser. A 240, 599 (1948).

${ }^{37}$ H. Kachkachi and D. A. Garanin, in Surface Effects in Magnetic Nanoparticles, edited by D. Fiorani (Springer, Berlin, 2005), p. 75.

${ }^{38}$ R. Yanes, O. Chubykalo-Fesenko, H. Kachkachi, D. A. Garanin, R. Evans, and R. W. Chantrell, Phys. Rev. B 76, 064416 (2007).

${ }^{39}$ M. Jamet, W. Wernsdorfer, C. Thirion, D. Mailly, V. Dupuis, P. Mélinon, and A. Pérez, Phys. Rev. Lett. 86, 4676 (2001); M. Jamet, W. Wernsdorfer, C. Thirion, V. Dupuis, P. Mélinon, A. Pérez, and D. Mailly, Phys. Rev. B 69, 024401 (2004).

${ }^{40}$ P. M. Déjardin, H. Kachkachi, and Yu. P. Kalmykov, J. Phys. D 41, 134004 (2008).

${ }^{41}$ I. S. Gradshteyn and I. M. Rizhyk, Table of Integrals, Series, and Products, 2nd ed. (Academic, New York, 1980). 